\title{
Frequency characteristics of accommodation in a patient with agenesis of the posterior vermis and normal subjects
}

Kenji Ohtsuka, Masahiro Sawa

\begin{abstract}
Aims-To clarify the cerebellar control of accommodation in humans, the frequency characteristics of accommodation were studied in a patient with agenesis of the posterior cerebellar vermis and four age matched normal subjects.

Methods-Magnetic resonance imaging of the brain of the 29 year old patient showed agenesis of the vermis and paravermis of lobules VIII-X and hypoplasia of the vermis and paravermis of lobules I-VII, the cerebellar hemisphere, and the cerebellar nuclei. The gain and phase lag of accommodative responses for sinusoidally modulated blur stimuli were calculated for the patient and four normal subjects. The blur stimuli consisted of predictable simple sinusoids of 3.0 dioptres at a frequency of $0.05,0.1,0.2,0.3,0.5$, or 1.0 Hz.

Results-The frequency characteristics of accommodative responses in the patient have a larger phase lag and a smaller gain at higher frequencies than those in the four normal subjects.

Conclusions-These findings suggest that the cerebellum contributes to the control of accommodation by improving the frequency characteristics at high frequencies.

(Br F Ophthalmol 1997;81:476-480)
\end{abstract}

Previous neurophysiological studies demonstrated that accommodative responses were evoked by electrical stimulation of the interpositus and fastigial nuclei in the cat. ${ }^{12}$ No responses could be evoked from the lateral cerebellar nuclei. Accommodative responses were also evoked by stimulation of the cerebellar cortex. ${ }^{1}$ The effective sites were distributed mostly in the vermis and paravermis of lobules VI, VII, and VIII, as well as the medial side of the paramedian lobule. The existence of both monosynaptic and disynaptic projections of the interpositus and fastigial nuclei to the parasympathetic oculomotor neurons in the cat and monkey has been demonstrated. ${ }^{3}{ }^{4} \mathrm{It}$ is likely that a paravermal-interpositus microcomplex and a vermal-fastigial microcomplex are involved in the control of accommodation. However, Westheimer and Blair ${ }^{5}$ found that unilateral cerebellar lobectomy affected neither accommodation nor pupillary movements in the monkey. The functional significance of the cerebellum in the control of accommodation remains unknown. The cerebellum is not likely to contribute to initiation of accommodation, but it is possible that it is involved in the modulation of parameters of accommodative responses.

It has been reported that the frequency characteristics of the pupillary light reflex for sinusoidally modulated light stimuli were impaired by cerebellectomy in the cat. ${ }^{67}$ The gain was reduced and the phase lag increased at high frequencies. It appears to show that the cerebellum contributes to the pupillary light reflex by improving the frequency characteristics of the reflex at high frequency. Therefore it seems likely that the cerebellum is related to the accommodation system in the same manner. However, no comparable study on accommodation has been reported.

In the present study, the frequency characteristics of accommodative responses for sinusoidally modulated blur stimuli were examined using a high speed infrared optometer in a patient with Joubert syndrome who showed agenesis of the posterior cerebellar vermis with normal intelligence. As normal controls, four age matched normal subjects were studied using the same paradigm.

\section{Materials and methods}

SUBJECTS

The patient was a 29 year old man with normal intelligence. $\mathrm{He}$ was referred to our clinic because of dimming of vision during a short period immediately after changing accommodation from distant to near vision. His visual acuity was $20 / 20$ in each eye. $\mathrm{He}$ was emmetropic and orthophoric. Slit lamp and funduscopic examinations showed no abnormalities. Visual fields were normal. His accommodative amplitude was 4.5 dioptres (D). Horizontal eye movements were recorded by direct current electro-oculography. The patient showed gaze evoked nystagmus at eccentric eye positions, downbeat nystagmus during down gaze, and rebound nystagmus. Saccadic eye movements were dysmetric, and the gain of pursuit eye movements was markedly reduced. Figure 1 shows magnetic resonance images of the patient's brain, which indicate agenesis of the vermis and paravermis of lobules VIII-X, and hypoplasia of the vermis and paravermis of lobules I-VII, the cerebellar hemisphere, and the cerebellar nuclei (the interpositus and fastigial nuclei), and expansion of the IVth ventricle. The brainstem and cerebral cortex appeared to be intact. The patient was
Accepted for publication 12 February 1997 

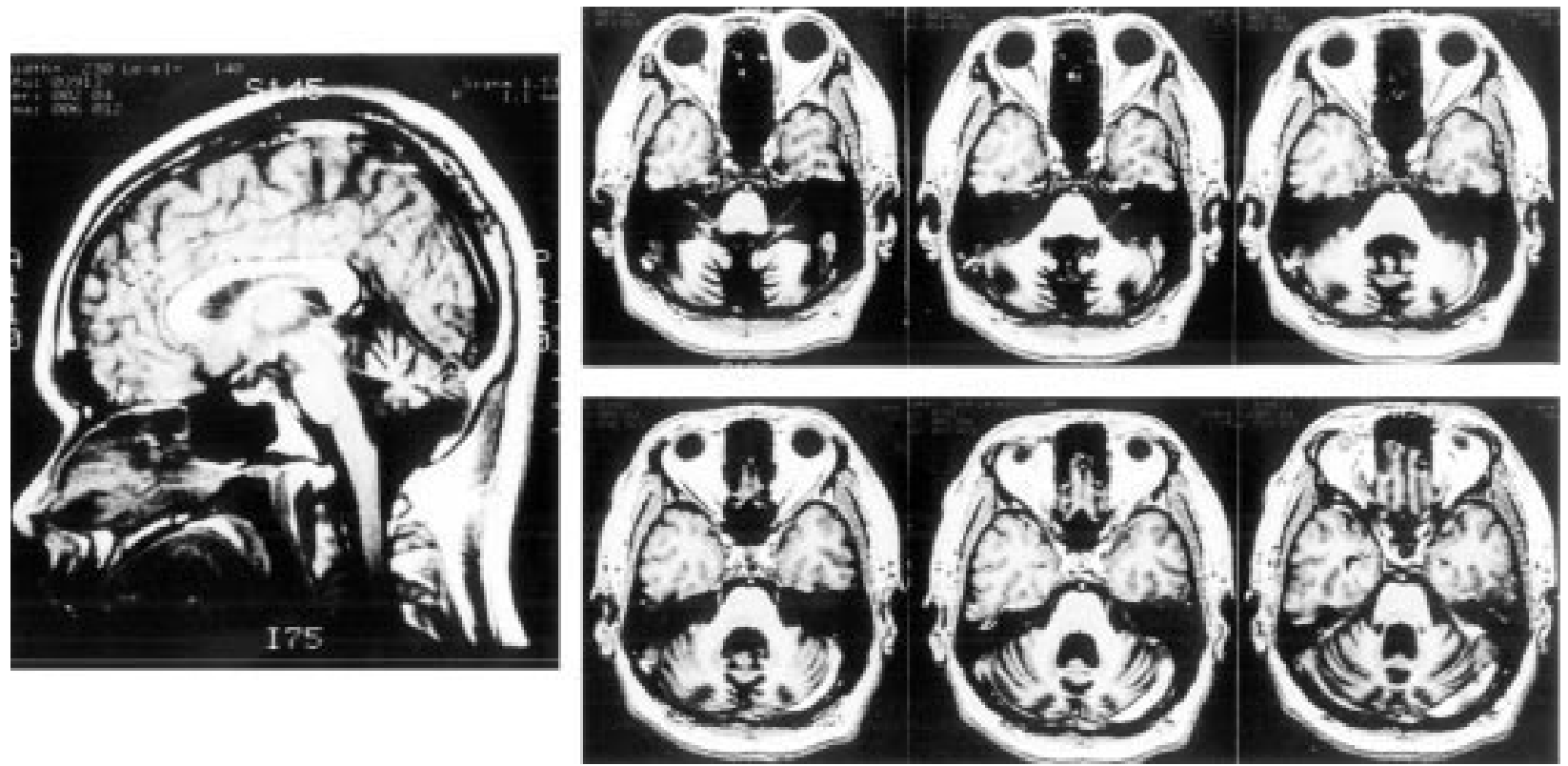

Figure $1 T 1$ weighted magnetic resonance images of the brain of the 29 year old patient showing agenesis of the vermis and paravermis of lobules VIII-X, and hypoplasia of the vermis and paravermis of lobules I-VII, the cerebellar hemisphere, and the cerebellar nuclei.

diagnosed as having Joubert syndrome or congenital agenesis of the cerebellar vermis. ${ }^{8-10}$ The patient had received no medication for two weeks before the experiment. Four age matched emmetropic and orthophoric volunteers were selected as normal controls. Their visual acuities were $20 / 20$ or better.

RECORDING PROCEDURES AND DATA ANALYSIS Accommodative responses of the right eye were continuously recorded with an infrared optometer (Nidek, model AR-1100). This system was basically analogous to that developed by Cornsweet and Crane. ${ }^{11}$ For accurate measurement, the ocular alignment of the right eye was continuously monitored with an infrared TV monitor mounted in the optometer. This system has a resolution of $0.01 \mathrm{D}$. The optometer had a target system for blur stimuli. The target was shaped like an asterisk, with eight black lines radiating in eight directions, $45^{\circ}$ apart, located at the centre of an illuminated field. The asterisk subtended an angle of $3^{\circ}$, and the width of each line was 40 minutes of arc. The dioptric distance of the target was controlled by a microcomputer. The target position and size are constant regardless of dioptric distance in this system. The blur stimuli consisted of predictable simple sinusoids of $3.0 \mathrm{D}$ at a frequency of $0.05,0.1,0.2$, $0.3,0.5$, or $1.0 \mathrm{~Hz}$. Amplitudes of accommodative responses were calibrated by inserting trial lenses of +1 and $+2 \mathrm{D}$ before the subject's eye, while the deflection of the recorder was noted. Calibrations were performed periodically throughout the experiment, and were found to be linear and consistent. During the experiment, the subjects were seated on a chair with the head and chin resting on a frame. Informed consent was obtained from the patient and the four normal subjects after the nature of the procedure had been explained. Tenets of the Declaration of Helsinki were fol- lowed, and institutional human experimentation committee approval was obtained.

Accommodative responses and target movements were recorded on magnetic tapes for subsequent computer analysis, using a data recorder. The data recorded on magnetic tapes were digitised by a computer at a sampling rate of $200 \mathrm{~Hz}$. To study the frequency characteristics of accommodation, the gain and phase lag of accommodative responses at each frequency were calculated for the patient and the normal subjects. In addition, Fourier analysis was performed to study frequency components of the waveform of accommodative responses in each subject.

\section{Results}

Figure 2 shows examples of accommodative responses of the patient and one of the four normal subjects at frequencies of $0.1,0.2$, and $0.3 \mathrm{~Hz}$. Waveforms of accommodative responses to the simple sinusoidal stimuli in the patient were relatively irregular compared with those in the normal subjects. Figure 3 shows results of Fourier analysis of accommodative responses in the patient and one of the four normal subjects. Accommodation components at frequencies corresponding to the stimulus frequencies were markedly smaller in the patient than in the normal subjects at all frequencies tested in this study. Accommodation components corresponding to the stimulus frequencies in the patient were about 50, 27 and $12 \%$ of those in the normal subjects at 0.1 , 0.2 , and $0.3 \mathrm{~Hz}$ respectively. In the normal subjects, amplitudes of frequency components corresponding to the stimulus frequencies were fairly constant at frequencies of less than 0.3 $\mathrm{Hz}$. Accommodation components at harmonic frequencies corresponding to the stimulus frequencies of 0.2 and $0.3 \mathrm{~Hz}$ were also observed in the normal subjects, but these were not detected in the patient. 

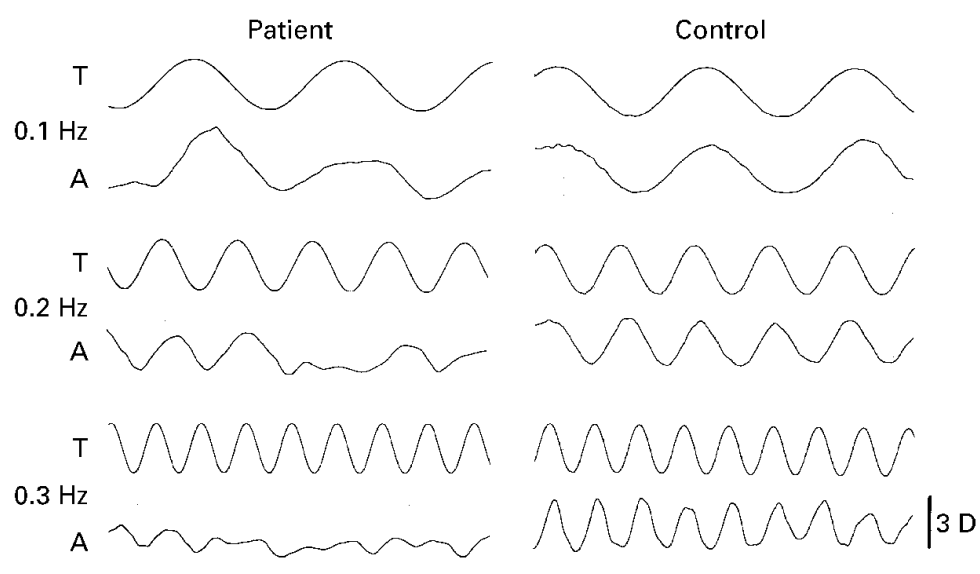

Figure 2 Examples of accommodative responses to simple sinusoidal blur stimuli at frequencies of $0.1,0.2$, and $0.3 \mathrm{~Hz}$ in the patient and a normal subject. $T=$ target movements; $A$ = accommodative responses.
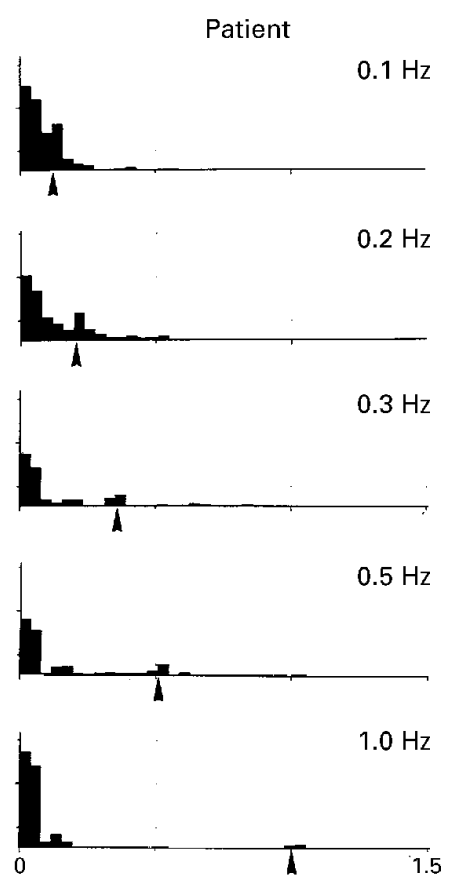

Frequency $(\mathrm{Hz})$
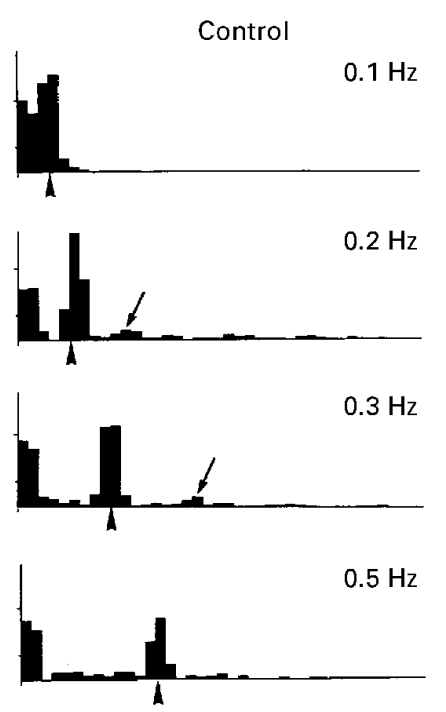

Frequency $(\mathrm{Hz})$

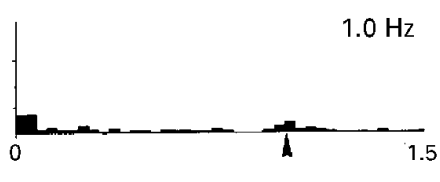

Figure 3 Examples of Fourier analysis of accommodative responses to simple sinusoidal stimuli at frequencies of $0.1,0.2,0.3,0.5$, and $1.0 \mathrm{~Hz}$ in the patient and a normal subject. Each arrowhead indicates the stimulus frequency. Arrows indicate accommodation components at harmonic frequencies.

Figure 4 shows the frequency characteristics of accommodative responses in the patient and the four normal subjects. The frequency characteristics in the normal subjects were fairly consistent. The phase lag of accommodative responses in the patient was larger than that in the normal subjects especially at higher frequencies. In the high frequency range $(>0.2$ $\mathrm{Hz}$ ) accommodative responses in the patient had a phase lag about 30-70 degrees larger than those in the normal subjects, although there was little difference in the phase lag between the patient and the normal subjects at low frequencies $(<0.1 \mathrm{~Hz})$. The gain of accommodative responses in the patient was smaller than that in the normal subjects at all frequen-
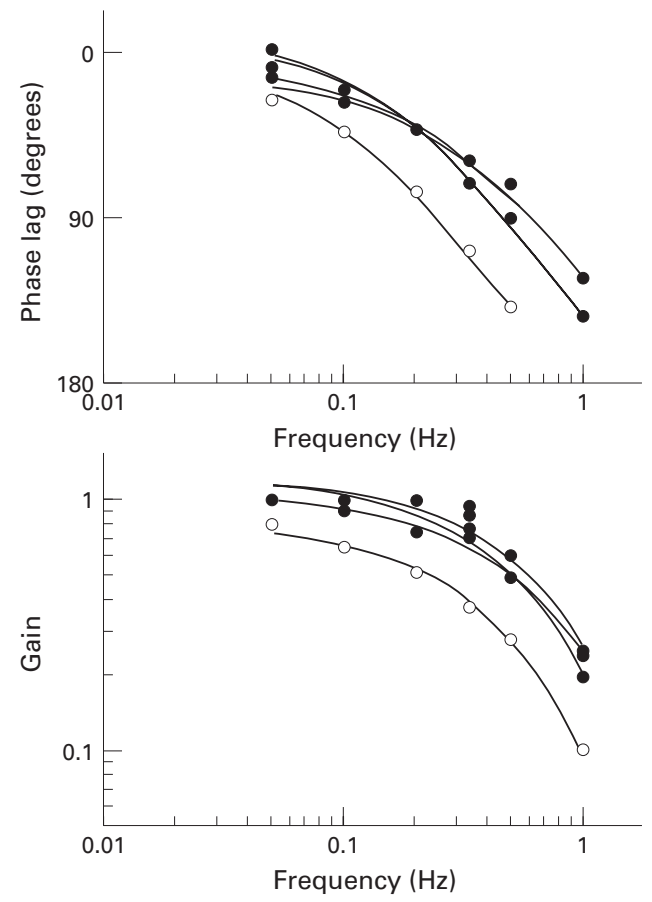

Figure 4 Frequency characteristics (phase lag and gain) of accommodative responses in the patient (open circles) and the four normal subjects (closed circles). Each regression curve is an exponential curve fitted by the method of least squares.

cies tested in this study. The difference in the gain ranged from 0.1 to 0.55 .

\section{Discussion}

The present findings suggest that the cerebellum contributes to accommodative responses by improving dynamic characteristics at high frequencies. Results of previous neurophysiological studies of the cat ${ }^{12}$ suggested that the vermis and paravermis of lobules VI-VIII, and the interpositus and fastigial nuclei are involved in the control of accommodation. The patient in the present study showed agenesis or marked hypoplasia of these portions of the cerebellum.

In a previous study on human subjects, accommodative responses were predictably controlled especially in the high frequency range of $0.3-1.0 \mathrm{~Hz} .{ }^{12}$ The predictable simple sinusoidal response has about a 30 degree smaller phase lag and $2 \mathrm{~dB}$ larger gain than the unpredictable multiple sinusoidal response. The patient in the present study exhibited a 30-70 degree larger phase lag and a $0.1-5.5$ (2-4 dB) smaller gain in predictable accommodative responses than the normal subjects. It is therefore probable that the predictable control of accommodation was impaired in the patient. These findings suggest that the cerebellum is involved in the predictable control of accommodation.

Results of previous studies have suggested that the lateral suprasylvian (LS) area, the cortical area surrounding the middle suprasylvian sulcus of the cat, is related to the control of lens accommodation. ${ }^{213} 14$ The LS area receives visual inputs. Some neurons in this area responded to changes in ocular disparity and 


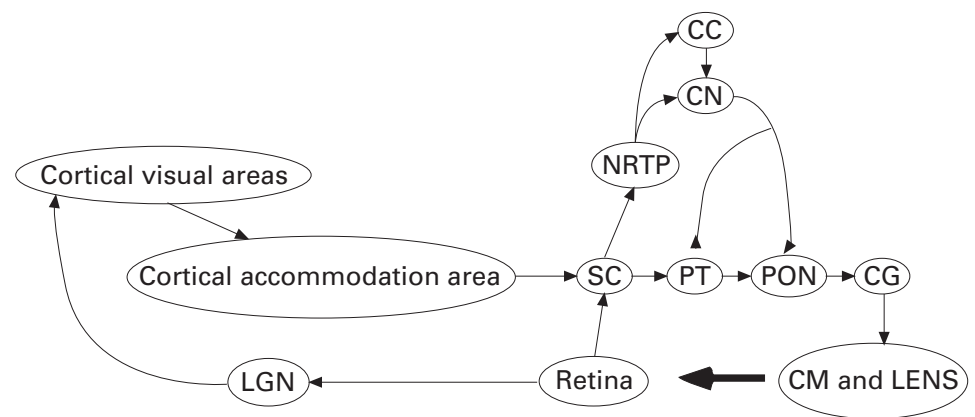

Figure 5 A model for the accommodation control system. The cortical accommodation area projects command signals (desired accommodation) to the SC. The SC projects to the PON through the PT. This is the main pathway of the accommodation system. The SC also projects to the cerebellum through the NRTP. The cerebellum sends fibres to the PT and the $P O N$. The cerebellum constitutes the side pathway of the accommodation control system. $C C=$ cerebellar cortex $; C G=$ ciliary ganglion $; C M=$ ciliary muscle $; C N=$ cerebellar nuclei; $L G N=$ lateral geniculate nucleus; NRTP = nucleus reticularis tegmenti pontis, $P O N=$ parasympathetic oculomotor neurons; $P T=$ pretectum; $S C=$ superior colliculus.

target size and to motion in depth, which are important visual cues for accommodation..$^{15-17}$ Some LS neurons also exhibited burst discharges preceding the onset of spontaneous accommodation. ${ }^{2}$ It is likely that these neurons have an important role in the control of accommodation. We have reported that, after injection of wheat germ agglutinin horseradish peroxidase into the low threshold area for evoking accommodative responses in the LS area of the cat, dense labelling of axon terminals was observed in the rostral portion of the ipsilateral superior colliculus (SC). ${ }^{18}$ Accommodative responses evoked by microstimulation of the LS area were almost abolished after injection of muscimol, an agonist of the inhibitory neurotransmitter $\gamma$ aminobutyric acid into the rostral SC. ${ }^{19}$ These findings suggest that accommodation related signals from the cortex project mainly to the rostral SC.

Accommodative responses were evoked by low current stimulation $(<20 \mu \mathrm{A})$ of a circumscribed area of the rostral SC, corresponding to the terminal portion of the descending projections from the cortical accommodation area in the LS area. ${ }^{20}$ The accommodation related area in the rostral SC is thought to project to the cerebellum through the medial part of the nucleus reticularis tegmenti pontis (NRTP) in the cat. ${ }^{21}$ Recently Gamlin and Clarke ${ }^{22}$ reported that neurons in the medial part of the NRTP exhibited discharges related to vergence and accommodation in the monkey. The medial NRTP has been reported to project to the vermis and paravermis of lobules VI-VIII and the interpositus and fastigial nuclei. ${ }^{23-27}$

The accommodation related area in the rostral SC also projects to the nucleus of the optic tract (NOT), the nucleus of the posterior commissure (NPC), and the mesencephalic reticular formation (MRF). ${ }^{20}$ These areas in the midbrain have been reported to be involved in the control of accommodation or vergence. ${ }^{28-31}$ The NOT and NPC send axons to parasympathetic oculomotor neurons. ${ }^{32-34}$ The interpositus nucleus sends axons to the NRTP, the pontine nuclei, the pretectum, the SC, and Edinger-Westphal's nucleus. ${ }^{45}{ }^{36}$ The fastigial nucleus also sends axons to the pretectum, the SC, the NRTP, and the pontine nuclei. ${ }^{22}{ }^{36-38}$
Figure 5 shows a schematic diagram of the accommodation control system including the cerebellum. The cortical centre of accommodation receives the visual input from the visual cortex, and sends axons to the rostral SC. The rostral SC projects to parasympathetic oculomotor neurons through the NOT and NPC. This is thought to be the main pathway of the accommodation system. The rostral SC also projects to the cerebellum through the NRTP. Cerebellar efferent fibres finally terminate in the premotor circuit in the midbrain. This pathway including the cerebellum is thought to be a side pathway of the accommodation control system. The cerebellar side pathway may be involved in the predictable control of accommodation and contribute to improving or adjusting parameters of accommodative responses. $^{39}$

This work was supported in part by a Grant-in-Aid for Science and Culture (04771362).

1 Hosoba M, Bando T, Tsukahara N. The cerebellar control of accommodation of the eye in the cat. Brain Res 1978;153:495-505.

2 Bando T, Yamamoto N, Tsukahara N. Cortical neurons related to lens accommodation in posterior lateral suprasylvian area in cats. $\mathcal{F}$ Neurophysiol 1984;52:879-91.

3 Hultborn H, Mori K, Tsukahara N. The cerebellar influence on parasympathetic neurons innervating intraocular muscles. Brain Res 1978;159:269-78.

4 Carpenter MB, Strominger NL. Cerebello-oculomotor fibers in the rhesus monkey. F Comp Neurol 1964;123:21130.

5 Westheimer G, Blair SM. Functional organization of primate oculomotor system revealed by cerebellectomy. Exp Brain Res 1974;21:463-72.

6 Tsukahara N, Kiyohara T, Ijichi Y. The mode of cerebellar control of pupillary light reflex. Brain Res 1973;60:244-8.

7 Ijichi Y, Kiyohara T, Hosoya M, Tsukahara N. The cerebelIjichi Y, Kiyohara T, Hosoya M, Tsukahara N. The cerebel-
lar control of the pupillary light reflex in the cat. Brain Res 1977;128:69-79.

8 Joubert M, Eisenring JJ, Robb JP, Andermann F. Familial agenesis of the cerebellar vermis. A syndrome of episodic hyperpnea, abnormal eye movements, ataxia, and retardation. Neurology 1969;19:813-25.

9 Sarnat HB, Alcala H. Human cerebellar hypoplasia. A syndrome of diverse causes. Arch Neurol 1980;107:300-5.

10 Lambert S, Kriss A, Gresty M, Benton S, Taylor D. Joubert syndrome. Arch Ophthalmol 1989;107:709-13.

11 Cornsweet TN, Crane HD. Servo-controlled infrared optometer. F Opt Soc Am [A] 1970;60:548-54.

12 Krishnan VV, Phillips S, Stark L. Frequency analysis of accommodation, accommodative vergence and disparity vergence. Vision Res 1973;13:1545-54.

13 Bando T, Tsukuda K, Yamamoto N, Maeda J, Tsukahara N. Cortical neurons in and around the Clare-Bishop area related with lens accommodation in the cat. Brain Res 1981;225:195-9.

14 Sawa M, Maekawa H, Ohtsuka K. Cortical area related to lens accommodation in cat. Jpn f Ophthalmol 1992;36:3719.

15 Toyama K, Kozasa T. Responses of Clare-Bishop neurons to three-dimensional movement of a light stimulus. Vision Res 1982;22:571-4.

16 Toyama K, Fujii K, Kasai S, Maeda K. The responsiveness of Clare-Bishop neurons to size cues for motion stereopsis. Neurosci Res 1986;4:110-28.

17 Toyama K, Komatsu Y, Kozasa T. The responsiveness of Clare-Bishop neurons to motion cues for motion stereopClare-Bishop neurons to motion
sis. Neurosci Res $1986 ; 4: 83-109$.

18 Maekawa H, Ohtsuka K. Afferent and efferent connections of the cortical accommodation area in the cat. Neurosci Res of the cortical accom

19 Ohtsuka K, Sato A. Descending projections from the cortical accommodation area in the cat. Invest Ophthalmol Vis Sci 1996;37:1429-36.

20 Sawa M, Ohtsuka K. Lens accommodation evoked by microstimulation of the superior colliculus in the cat. Vision Res 1994;34:975-81.

21 Sato A, Ohtsuka K. Projections from the accommodationrelated area in the superior colliculus of the cat. 7 Comp Neurol 1996;367:465-76.

22 Gamlin PDR, Clarke RJ. Single-unit activity in the primate nucleus reticularis tegmenti pontis related to vergence and nucleus reticularis tegmenti pontis related to vergence and

23 Noda H, Sugita H, Ikeda Y. Afferent and efferent connections of the oculomotor region of the fastigial nucleus in the macaque monkey. F Comp Neurol 1990;302: 330-48. 
24 Yamada J, Noda H. Afferent and efferent connections of the oculomotor cerebellar vermis in the macaque monkey. $f$ oculomotor cerebellar vermis
Comp Neurol 1987;265:224-41.

25 Hoddevik GH. The projection from the nucleus reticularis tegmenti pontis onto the cerebellum in the cat. Anat Embryol (Berl) 1978;153:227-42.

26 Brodal P. The projection from the nucleus reticularis tegmenti pontis to the cerebellum in the rhesus monkey. Exp Brain Res 1980;38:29-36.

27 Kawamura K, Hashikawa T. Projections from the pontine nuclei proper and reticularis tegmental nucleus onto the cerebellar cortex in the cat. An autoradiographic study. $\mathcal{F}$ Comp Neurol 1981;201:395-413.

28 Judge SJ, Cumming BG. Neurons in the monkey midbrain with activity related to vergence eye movement and accommodation. I Neurophysiol 1986;55:915-30.

29 Mays LE. Neural control of vergence eye movements: convergence and divergence neurons in midbrain. I Neurovergence and divergence newr
physiol. 1984;51:1091-1108.

30 Bando T, Tsukuda K, Yamamoto N, Maeda J, Tsukahara N. Physiological identification of midbrain neurons related to lens accommodation in cats. F Neurophysiol 1984;52:870-8.

31 Konno S, Ohtsuka K. Accommodation-related area in the pretectal nuclei of the cat. Invest Ophthalmol Vis Sci 1995;36:694
32 Berman, N. Connections of the pretectum in the cat. 7 Comp Neurol 1977; 174:227-54

33 Graybiel AM, Hartwieg EA Some afferent connections of the oculomotor complex in the cat: an experimental study with tracer techniques. Brain Res 1974;81:543-51.

34 Buttner-Ennever JA, Cohen B, Horn AKE, Reisine H. Pretectal projections to the oculomotor complex of the monkey and their role in eye movements. F Comp Neurol 1996;366:348-59

35 McCrea RA, Bishop GA, Kitai ST. Morphological and elecrophysiological characteristics of projection neurons in the nucleus interpositus of the cat cerebellum. F Comp Neurol 1978; 181:397-402.

36 Sugimoto T, Mizuno N, Uchida K. Distribution of cerebellar fiber terminals in the midbrain visuomotor areas: an autoradiographic study in cat. Brain Res 1982;238:353-70.

37 Batton RR III, Yayeraman A, Ruggiero D, Carpenter MB. Fastigial efferent projections in the monkey: an autoradioFastigial efferent projections in the monkey: an
graphic study. $\mathcal{F}$ Comp Neurol 1977;174:281-306.

38 Hirai T, Onodera S, Kawamura K. Cerebellotectal projections studied in cats with horseradish peroxidase or tions studied in cats with horseradish peroxidase or
tritiated amino acid axonal transport. Exp Brain Res 1982; tritiated

39 Ito M. The cerebellum and neural control. New York: Raven Press, 1984. 